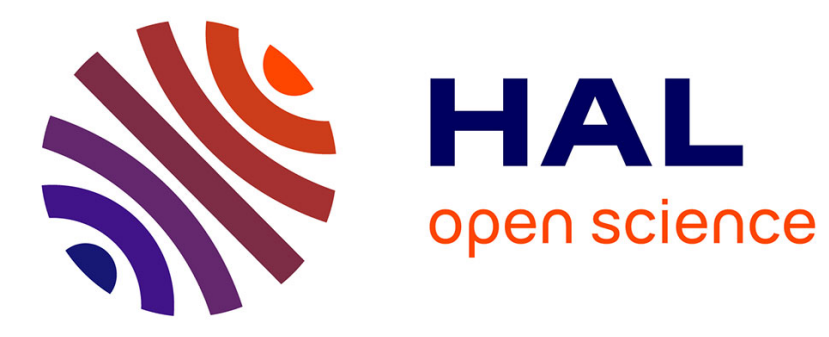

\title{
Subpicomolar Iron Sensing Platform Based on Functional Lipid Monolayer Microarrays
}

Ahmad Kenaan, Tuyen D. Nguyen, Herve Dallaporta, Jean-Manuel Raimundo, Anne M. Charrier

\section{To cite this version:}

Ahmad Kenaan, Tuyen D. Nguyen, Herve Dallaporta, Jean-Manuel Raimundo, Anne M. Charrier. Subpicomolar Iron Sensing Platform Based on Functional Lipid Monolayer Microarrays. Analytical Chemistry, 2016, 88 (7), pp.3804-3809. 10.1021/acs.analchem.5b04834 . hal-01459052

\section{HAL Id: hal-01459052 https://hal.science/hal-01459052}

Submitted on 19 Mar 2019

HAL is a multi-disciplinary open access archive for the deposit and dissemination of scientific research documents, whether they are published or not. The documents may come from teaching and research institutions in France or abroad, or from public or private research centers.
L'archive ouverte pluridisciplinaire HAL, est destinée au dépôt et à la diffusion de documents scientifiques de niveau recherche, publiés ou non, émanant des établissements d'enseignement et de recherche français ou étrangers, des laboratoires publics ou privés. 


\title{
Sub-picomolar iron sensing platform based on
}

\section{functional lipid monolayer microarrays}

\author{
Ahmad Kenaan, ${ }^{\dagger, \uparrow}$ Tuyen D. Nguyen, ${ }^{\ddagger}, \uparrow$ Hervé Dallaporta, ${ }^{\dagger}$ Jean-Manuel \\ Raimundo, ${ }^{\dagger}$ and Anne M. Charrier*, ${ }^{*}$ \\ Aix-Marseille Université, CNRS, CINaM-UMR 7325, Marseille 13288, France, and \\ Institute for Microelectronic Technology and Information, NACENTECH, Ha Noi, Vietnam \\ E-mail: charrier@cinam.univ-mrs.fr
}

\begin{abstract}
We report herein the fabrication of novel microarrays based on air-stable functional lipid monolayers over silicon using a combination of e-beam lithography and lift-off. We demonstrate these microarrays can be use as ultra-sensitive platform for Kelvin probe force microscopy in sensing experiments. Specificity of the detection is given by the functional group grafted at the lipid head-group. The arrays developed for the detection of ferric ions, $\mathrm{Fe}^{3+}$, using a $\gamma$-pyrone derivative chelator, demonstrate sub-picomolar limit of detection with high specificity. In addition, the technique takes advantage of the structure of the array with the silicon areas playing the role of reference for the measurement, and we determine critical pattern dimensions below which the probe size/shape impacts the measured results.
\end{abstract}

*To whom correspondence should be addressed

†Aix-Marseille Université, CNRS, CINaM-UMR 7325, Marseille 13288, France

${ }_{\ddagger}^{\ddagger}$ Institute for Microelectronic Technology and Information, NACENTECH, Ha Noi, Vietnam

ฯ Contributed equally to this work 
Microarrays of lipid bilayers and monolayers find extensive applications in the fields of biology and medical diagnosis ${ }^{1-4}$. Lipid layers constitute very interesting model system of biological membranes ${ }^{5,6}$ wherein proteins can be embedded ${ }^{7}$ and used for instance to study cell-cell interactions mechanisms such as the role of conformational changes or organization of receptors in intercellular signaling processes ${ }^{8}$. Other studies requiring high throughput such as measuring the interaction between lipid membranes and proteins or drug screening also constitute promising applications of these microarrays ${ }^{9}$.

Given the tremendous potential of such lipid layer microarrays, miscellaneous techniques based on diffusion barriers ${ }^{10-13}$, micro-contact printing ${ }^{1,14}$, non-contact printing, ${ }^{10,15}$, optical lithography ${ }^{16,17}$, and lift-off ${ }^{18,19}$ are currently used to create patterned substrates of lipid monolayers or bilayers. More recently surface modification of teflon has been demonstrated using e-beam lithography offering hindered areas that favor lipid adsorption and layer formation with areas of nanometric dimensions ${ }^{20}$. Similarly ink-jet printing ${ }^{15}$ or spotting techniques, ${ }^{10,21}$ alone or combine to aforementioned patterning techniques allow the formation of arrays of different molecules for multiplexed experiments. All these techniques are suitable for high throughput microarrays fabrication.

Growing interest in lipids as amphiphilic building block materials relies on their capability to form supported versatile platforms as monolayers, bilayers or multilayers ${ }^{22-24}$. Layers are usually obtained using either the Langmuir-Blodgett technique ${ }^{25-27}$ in which the lipids are prearranged in a monolayer at the air/water interface prior to transfer on the substrate or the lipid vesicle fusion technique which consists in putting a drop containing small unilamellar lipid vesicles on top of the substrate and wait for the vesicles to rupture as they get into contact with the substrate ${ }^{28,29}$. Both methods lead to the formation of well organized and dense monolayers on hydrophobic substrates and bilayers on hydrophilic's. For microarrays, the lipid vesicle method is the most suitable and commonly accepted one as it is adapted to high throughput array fabrication techniques.

Within this work we focus on the development of self-assembled functionalized lipid 
monolayer microarrays that can be used as an efficient sensing platform for the detection of specific ions using Kelvin probe force microscopy (KPFM). KPFM was very recently used to detect low concentrations of metal ions adsorbed on a substrate ${ }^{30,31}$. Along this work we used the vesicle fusion deposition technique coupled to electronic lithography and lift-off to generate our lipid microarrays. Moreover in order to ensure greater stability of the self-assembled monolayers, we performed 2D-polymerization within the plane of the monolayers following a procedure developed in our group. This step is of crucial importance as it enhances the stability of the device over long period of time when exposed to air or solvents ${ }^{32,33}$.

KPFM constitutes an extremely surface sensitive technique that measures the contact potential difference (CPD) between a conductive tip and a surface to analyze. ${ }^{34} \mathrm{CPD}$ is not only dependent on the nature of the sample and tip through their work function but also on the presence of contamination or adsorbate on their surfaces. Any adsorption of charges will inevitably induce a surface potential change and consequently a CPD change ${ }^{35-38}$. In principle the work function of the tip is fixed and known and might serve as reference. However, tip surface properties might evolve over time, leading to incoherent measurements. To prevent that to happen we propose to take advantage of the microarray geometry to create the sensing areas juxtaposed to areas of neat silicon playing the role of reference. Measuring the CPD difference between the lipid modified areas and the silicon areas will give rise to a direct measure of the surface potential change at the lipid interface.

As a proof of concept, we report in this manuscript a microarray sensor that was developed for the detection of ferric ions. 
Iron ions species play diverse and major roles in many important metabolic processes, biological materials and environmental samples. Although many analytical detection techniques of iron now exist only a few approaches have been successfully developed to quantify the different oxidation states of iron species in a sample ${ }^{39,40}$ and they usually have limited limit of detection. Speciation between $\mathrm{Fe}^{2+}$ and $\mathrm{Fe}^{3+}$ is one of the major issues in detecting methemoglobin in blood, in understanding the biochemical cycling in cell metabolic pro$\operatorname{cesses}^{30,41,42}$ as well as in the ocean or atmosphere for which these ions are involved in many processes including redox and radical chain reactions. ${ }^{43}$ Thus the development of sensors being able to detect and quantify small amounts of iron ions in a solution or in a biological sample is therefore highly relevant. All our detection tests have realized in ionic aqueous solutions. We demonstrate high sensitivity and specificity with a limit of detection in the sub-picomolar range.

\section{EXPERIMENTAL SECTION}

\section{Micro-patterning of MMA/PMMA bilayer}

Micro-patterning of MMA/PMMA (MonoMethylAcrylate/PolyMetaMethylAcrylate, SigmaAldrich) bilayer was realized in a clean-room facility (PLANETE, CT-PACA, Marseille, France) using standard procedures and electron-beam lithography system (Pioneer system from Raith, GMBH, Germany) as the radiation source for exposure. (100) silicon samples were cleaned with oxygen plasma, dipped $2 \mathrm{~min}$ in $2 \% \mathrm{HF}$ to remove silicon oxide and dehydrated 5 min at $180^{\circ} \mathrm{C}$. MMA and PMMA were consecutively spin coated on the sample (4000 RPM for 1 minute) and baked (5 minutes at $180^{\circ} \mathrm{C}$ ). MMA/PMMA bilayer was then exposed following a pre-designed pattern with e-beam lithography system (Beam current: $0.05 \mathrm{nA}$, area dose: $110 \mu \mathrm{C} / \mathrm{cm}^{2}$ ) and developed 1 min with a 1:3 MiBK:IPA mixture (Methyl isobutyl ketone, Isopropyl acetate, Sigma Aldrich). Samples were finally rinsed with IPA and blown dried with nitrogen. At this stage patterns of MMA/PPMA bilayer are present on the silicon surface. In the following, these patterns serve as mask for the formation of 
the lipid monolayer array following the under-mentioned protocol. After lipid deposition, MMA/PMMA bilayer is finally etched off by sonication in MPR 1165 (Shipley) solvent at $40^{\circ} \mathrm{C}$ for $1 \mathrm{~min}$, hence revealing a microarray of lipid monolayer.
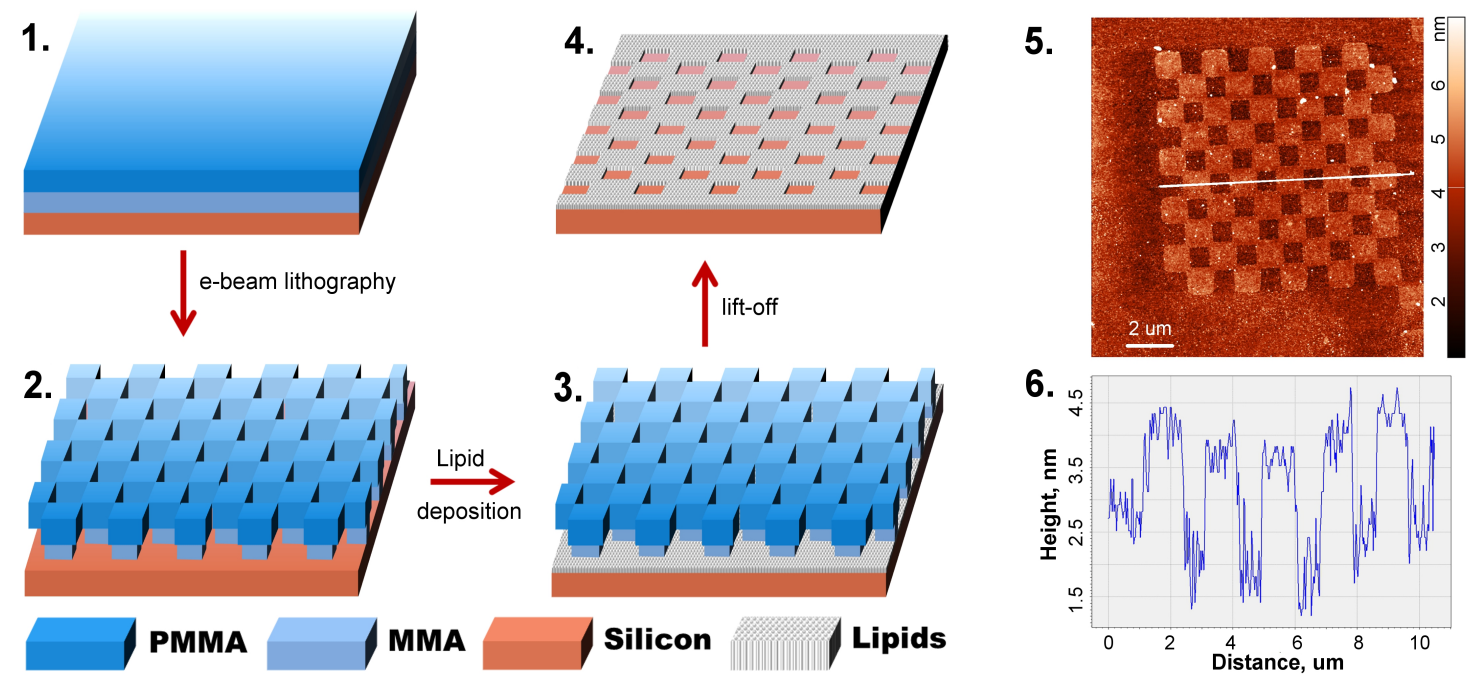

Figure 1: Lipid monolayer microarrays fabrication process: 1. Spin coating of MMA and then PMMA on silicon substrate. 2. Patterning of MMA/PMMA bilayer using e-beam lithography and development of exposed area. 3. Patterned MMA/PMMA bilayer is used as a mask for the formation in the open areas of lipid monolayers on silicon using the vesicle fusion technique. 4. Lift-off of remaining PMMA/MMA using MPR-1165 solvent. 5. AFM topographic image of an array of lipid monolayers on silicon with $1 \times 1 \mu \mathrm{m}^{2}$ square patterns. Lightest areas correspond to the lipids and darker to the silicon. The array cross-section shown in 6. indicates a lipid monolayer thickness of $\sim 2.2 \mathrm{~nm}$.

\section{Lipid monolayer fabrication}

Lipid monolayers are obtained using the well-known vesicle fusion method ${ }^{44,45}$. This method combined to ink jet printer or spotting techniques allows the fabrication of array platforms. Stock solutions of (1,2-bis-(10,12-tricosadiynoyl)-sn-glycero-3-phosphocholine lipids (DC8,9PC, Avanti Polar Lipids) at $1 \mathrm{mg} / \mathrm{mL}$ in chloroform, were prepared and stored at $-20^{\circ} \mathrm{C}$ prior use. Vesicles were prepared according to the following procedure: $50 \mu \mathrm{L}$ of a stock solution was smoothly heated at $40^{\circ} \mathrm{C}$ to ensure complete evaporation of chloroform then diluted in $200 \mu \mathrm{L}$ of DI water. To guaranty the formation of well-defined and small unil- 
amellar vesicles, the solution was sonicated for 15 minutes and repeatedly extruded through a polycarbonate membrane with $100 \mathrm{~nm}$ pores. Prior the vesicle deposition, the patterned sample (Fig. 1(3)) is dipped in a 2\% solution of HF in ethanol to remove native silicon oxide from the uncovered areas. Drops of lipid vesicles solution are then deposited on the patterned area at room temperature. The sample is then cooled down at $10^{\circ} \mathrm{C}$ for $5 \mathrm{~min}$. This process induces lipid vesicle condensation at the surface of the silicon and leads to the fast formation of a dense monolayer. The temperature is then slowly increased at a rate of $1^{\circ} \mathrm{C} / \mathrm{min}$ up to $35^{\circ} \mathrm{C}$ and the sample rinsed with clear water while maintaining the sample immersed. At this stage the monolayer is stable on the surface. To guaranty air-stability over a long period of time, the monolayer is stabilized, within the plane of the layer, by radical polymerization upon addition of $0.1 \%$ of $2,2^{\prime}$-Azobis(2-methylpropionamidine) dihydrochloride (AAPH, Sigma-Aldrich). The temperature is then slowly increased up to $43^{\circ} \mathrm{C}$ and left for 10 min. The chip is finally slowly cooled down to $25^{\circ} \mathrm{C}$ and carefully rinsed with DI water.

\section{Imaging and Kelvin probe force measurements}

An NTEGRA atomic force microscope from NT-MDT was used for both imaging and Kelvin probe force microscopy. Imaging was performed in tapping mode and in air using HQ/NCS35 tips with a spring constant of $\sim 5.4 \mathrm{~N} / \mathrm{M}$ (Mikromash). Amplitude modulation KPFM (AM-KPFM) experiments were realized using DPE18 tips (Mikromash) with the following characteristics: spring constant: $\sim 3.5 \mathrm{~N} / \mathrm{m}$, radius: $40 \mathrm{~nm}$, length: $15 \mu \mathrm{m}$, cone angle $40^{\circ}$, and cantilever width and length of $30 \mu \mathrm{m}$ and $350 \mu \mathrm{m}$ respectively.

To seperate the topographic signal from the surface potential, KPFM was performed using a two-pass mode. For each line of the resulting image, surface topography imaging is first performed, the tip is then widthdrawn from the surface by $10 \mathrm{~nm}$ for KPFM measurement. In all our experiments, the sample was connected to the ground and the tip polarized. 


\section{RESULTS AND DISCUSSION}

\section{Fabrication of lipid microarrays}

Self-assembled lipid monolayers arrays were fabricated using e-beam lithography associated to the lift-off technique as depicted in Fig. 1. A pattern constituted by a double layer of MMA/PMMA resist was first made starting from consecutive spin-coating of MMA and PMMA layers on a clean silicon surface. As a result of e-beam exposure, undercut MMA is obtained, therefore enlarging the exposed area and favoring ulterior lift-off. The patterned resist is then used as a mask for lipid deposition.

Commercially available DC8,9PC lipids have been specifically selected for having two acetylenic groups in each of their fatty-acid chains. The acetylenic groups are of importance as they ensure, after a polymerization process, higher mechanical stability of the selfassembled monolayer ${ }^{46}$. Firstly, lipid monolayer formation is obtained using the vesicle fusion technique following the procedure described in the materials and methods section. Consecutive free radical activated polymerization process was applied on the freshly deposited lipids leading to the formation of a monolayer which is stable enough to handle chemical lifting of the resists using the resist remover MPR 1165 and rinsing with water or solvents ${ }^{46}$. After lift-off the resulting lipid areas have a thickness of $\sim 2.2 \mathrm{~nm}$ as determined from AFM imaging measurements. Arrays constituted of $10 \times 10$ square patterns with side sizes of $1 \mu m$ and $3 \mu \mathrm{m}$ surrounded by $10 \times 10 \mu m^{2}$ and $30 \times 30 \mu m^{2}$ silicon regions respectively (Array 1 and Array 3) were fabricated (Fig. 1).

\section{$\mathrm{Fe}^{3+}$ ions detection: Synthesis of $\gamma$-pyrone chelator}

In microarray chip sensors, specific surface functionality is a required feature for effective detection specificity. We have demonstrated in a recent work the possibility to chemically engineer the lipid head-groups in order to bind a specific function ${ }^{47,48}$. The first step consists in removing the phosphocholine end-group by reacting the $\mathrm{DC} 8,9 \mathrm{PC}$ with a phospholipase $\mathrm{C}$ 
(Sigma-Aldrich) offering quantitatively the corresponding alcohol. Subsequent condensation with an appropriate functional group is then relatively straight-forward. In addition, this method brings high versatility to our system as the alcohol-lipid can be considered as a building-block lipid molecule.

In the specific case of ferric ions detection, we have developed a $\gamma$ pyrone derivative chelator synthesized from commercially available kojic $\operatorname{acid}^{47}$. The later is grafted to the lipid-alcohol using standard procedures. The $\gamma$-pyrone is the active component of the Ferriprox (C) (Apotex Europe) a commercial iron chelator used for the treatment of patients with transfusional iron overload due to thalassemia syndromes. $\gamma$-pyrones form five-membered chelate rings with iron in which the metal is coordinated by two vicinal oxygen atoms leading to neutral and stable 3:1 complexes.

This molecule was therefore already proven to be a good chelator of iron.

In our work we started from this commercially available molecule that we modified in order to bind it to the lipids. We have shown that ones bound to the lipids the chelator affinity towards ferrous ions drops while it remains several orders of magnitude higher for ferric ions ${ }^{48}$. Our final $\gamma$-pyrone modified lipid monolayer is hence expected to have high affinity towards ferric ions. To verify this point, specificity measurements were realized by exposing the Arrays to solutions of competing ions such as $\mathrm{Fe}^{2+}, \mathrm{Cu}^{2+}, \mathrm{Pb}^{2+}, \mathrm{Al}^{+}$, and $\mathrm{K}^{+}$.

Both the synthesis of the above modified lipid and the results of specificity measurements are described and shown in the supporting information file.

\section{KPFM imaging of lipid microarray}

Kelvin probe images of Array 1 and Array 3 of engineered lipid monolayers are shown in Fig. 2 (a) and (b). These images provide the CPD value measured between the AFM tip and the silicon substrate. The presence of lipid layer adsorbate on top of the silicon areas impacts dramatically this value. Lipid areas, darker grey, display more negative CPD values with respect to silicon areas. Surprisingly the CPD difference between lipid, $C P D_{L i p}$ and 

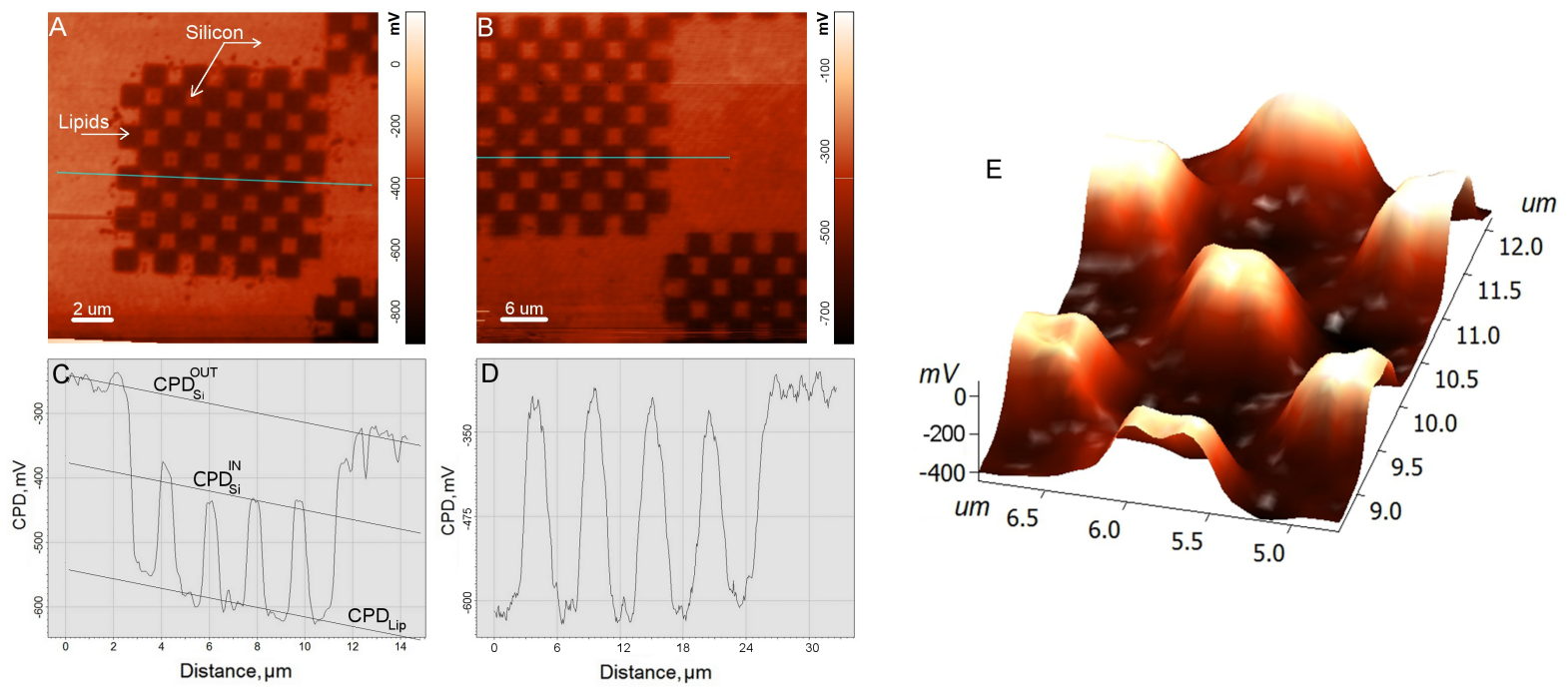

Figure 2: $\mathbf{A}$ and $\mathbf{B}$ : KPFM images of microarrays with $1 \times 1 \mu m^{2}$ (Array1) and $3 \times 3$ $\mu m^{2}$ (Array3) square patterns respectively. Darkest areas corresponds to the lipids and lightest to the silicon. $\mathbf{C}$ and $\mathbf{D}$ : Corresponding cross-sections give the contact potential difference $(\mathrm{CPD})$ between the tip and the measured area. $C P D_{S i}^{O U T}$ and $C P D_{S i}^{I N}$ correspond to the CPD values of silicon outside and inside the patterned zone. E: Three-dimensional KPFM image of the microarray within the patterned zone (The white spots in the baseline areas are artefacts of the 3D graphing software).

silicon, $C P D_{S i}$, areas varies with the size of the patterns. Similarly the CPD values of silicon areas within, $C P D_{S i}^{I N}$, and outside, $C P D_{S i}^{O U T}$, the patterned regions are different. This effect appears more pronounced for Array 1 as observed from the cross-section curves in Fig. 2 (c) and (d). The corresponding $\Delta(C P D)$ values are indicated in Table 1. Additionally, crosssection profiles of corresponding lipid and silicon areas surprisingly display conic-like shapes in place of expected plateaus (Fig. 2 (e)).

Table 1: Contact potential differences between the different areas of the arrays.

\begin{tabular}{lccc}
\hline & & & \\
& $\Delta C P D_{\text {Si-Lip }}^{I N}$ & $\Delta C P D_{\text {Si-Lip }}^{\text {OUT }}$ & $\Delta C P D_{\text {Si }}^{\text {OUT-IN }}$ \\
\hline $1 \times 1 \mu m^{2}$ & $180 \pm 4$ & $300 \pm 5$ & $122 \pm 7$ \\
$3 \times 3 \mu m^{2}$ & $324 \pm 6$ & $330 \pm 5$ & $5 \pm 5$ \\
\hline CPD in mV & & &
\end{tabular}

To have more insight and understand the importance of the patterns dimensions, one need to consider the electric field distribution between tip and surface during KPFM measurement. 


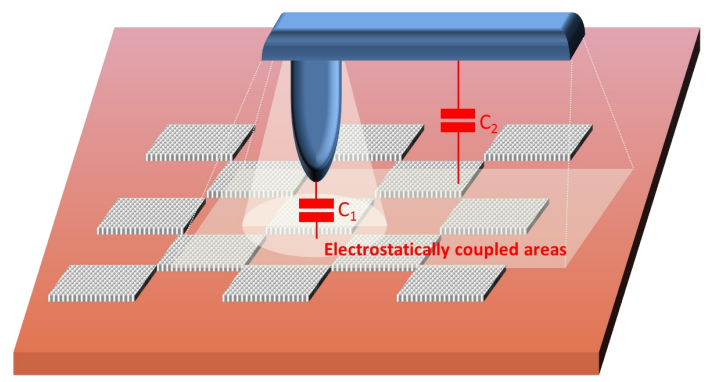

Figure 3: Scheme depicting the electrostatic coupling between the probe and the surface below. Measured CPD values correspond to an average of all the potentials on the surface weighted by the derivatives of the probe/surface regions capacitances.

It was demonstrated that the magnitude of the measured electric potential critically depends on the geometry of the tip, on the size and surrounding of the measured pattern and that its resolution and accuracy are defined by the electrostatic coupling between the tip and the different surface regions ${ }^{49}$. Therefore measured KPFM potential corresponds to an average of all potentials at the surface, the derivatives of the capacitances between the tip and surface being the weighting factors ${ }^{34,50}$. Considering these points, it is easy to explain the impact of the patterns size on the measured surface potentials and that for small size patterns, the tip shape might have an even stronger impact (Fig. 3). Hence, the minimum dimensions of the pattern areas must be chosen in order to neglect the geometric parameters. In our system, this critical size seems to be $3 \times 3 \mu m^{2}$ for which the maximum value of $C P D_{S i}^{I N}$ obtained in the center of the pattern is equal to $C P D_{S i}^{O U T}$.

\section{KPFM sensing experiments}

In a typical sensing experiment, the CPD of the modified lipid self-assembled monolayer is expected to vary linearly upon exposure to solutions of $\mathrm{Fe}^{3+}$. The CPD difference between the exposed lipid areas and silicon substrate can then be used to determine the surface potential change, $\Psi$, induced by the chelation of $\mathrm{Fe}^{3+}$ by the lipids and their stabilization with 2 Kojic acid molecules (Eq. 1) provided that the substrate surface property remains unchanged after exposure, i.e., unspecific adsorption is avoided. To 
limit such drawbacks, we have applied a peculiar protocol that combines both several thorough water rinsing and surface drying after each exposure. Kelvin Probe measurements are subsequently realized in air. Between two measurements, decomplexation of $\mathrm{Fe}^{3+}$ with the chelator is obtained upon rinsing the sensor with a base solution.

$$
\Psi_{\text {ions }}=\left(C P D_{\text {ExpLip }}-C P D_{S i}\right)-\left(C P D_{\text {Lip }}-C P D_{S i}\right)
$$

The measured change in CPD after complexation of the lipid head-groups with the Ferric ions and 2 Kojic acid molecules arises from a combination of factors: 1- a change of the dielectric properties of the lipids at their end-group region. These head-groups are most certainly rearranging after capture of the ions due to the coulombic repulsions between the ions. This effect is expected to be relatively small and might induce a positive or negative CPD change depending on whether it improves or damages the dielectric property of the layer. ${ }^{51,52} 2$ - a change in the surface charge density due to the capture of Ferric ions is certainly the stronger effect and should induce a positive surface potential change proportional to the amount of captured charges. This effect is expected to be enhanced due to the high dipole moment of the two lipid carbonyl chains. 3- with its highly delocalized electron cloud, the presence of the Kojic acid molecules is expected to induce a negative change of the CPD value. ${ }^{53}$ The final $\mathrm{CPD}_{\text {ExpLip }}$ change that we measure integrates all these components.

To improve the signal to noise ratio, we took advantage of the microarray structure and averaged for both silicon and lipid the different areas indicated with a green and red spot respectively on Fig. 4 (A). We considered only the areas having similar surrounding, i.e. excluding the areas located on the side on the large pattern area. The result of averaging is shown in Fig. 4 (B). The CPD difference between silicon and lipid area was then obtained by fitting the profile line taken along the center of the two areas with a sigmoid curve (Fig. 4 $(\mathrm{C}))$.

For both Array 1 and Array 3, Fig. 5 reports the dependence of $\Psi$ with respect to the ferric ions concentration. These results were obtained from two sets of measurements realized 

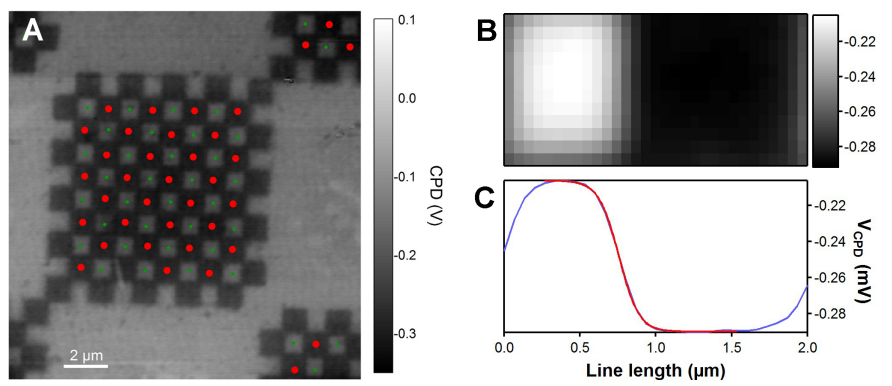

Figure 4: (A) KPFM image of an Array 1 microarray. Lipid areas (with red spot) and silicon areas (with green spots) are averaged (B). (C) Cross section of the averaged image along the blue line.

on two different samples and using different tips. In both cases, $\Psi$ varies linearly with the logarithm of the ferric ions concentration in the range $100 \mathrm{fM}$ to $1 \mu \mathrm{M}$ with $\psi$ reaching $100 \mathrm{mV}$ and $150 \mathrm{mV}$ at $1 \mu \mathrm{M}$ for Array 1 and Array 3 respectively. Above a sub-linearity suggests that the probe chelators bound to the lipids must be nearly saturated with ions. Both arrays demonstrate very high sensitivity with a limit of detection at $1 \mathrm{pM}$. 
Surprisingly, the dynamics of Array 1 and Array 3 are different. Array 1 curve presents a slope of $11.9 \mathrm{mV} / \log [$ ions] while Array 3 of $19.5 \mathrm{mV} / \log [$ ions]. Additionally, the values obtained for Array 1 show important deviations from the "ideal" linear curve obtained from fitting the data. These observations corroborate the above discussion on the measured CPD potential. This tends to decrease the CPD difference between silicon and layer areas. This also means that depending on the orientation of the probe with respect to the surface small variations of the $\mathrm{CPD}$ values can be measured. In figure 5, the orientation between the probe and the surface was randomly displayed after each measurement therefore explaining the non-linearity of the Array 1. Therefore, in order to be reproducible, the dimensions of the patterns must be sufficiently large so that the electrostatic coupling of the surface with the probe does not change with their relative orientation. With pattern dimension of $3 \times 3 \mu m^{2}$, Array 3 shows much better linearity. In the section "KPFM imaging of lipid microarrays" we have previously discussed that the $\mathrm{CPD}$ values measured on silicon areas outside, $C P D_{S i}^{O U T}$ and inside, $C P D_{S i}^{I N}$, the patterns have very close values, therefore suggesting that these areas are sufficient large to minimize the averaging effect. Similar conclusion can be concluded for the sensing experiments and the values of the surface potential measured in the center of the Array 3 areas after ion exposure can be considered as the real surface potential. Sensitivity performances of the sensors can be determined using the Nernst Equation (Eq. 2). This relation is routinely used in electrochemistry and relates the potential of the sensitive ion electrode (ISE) after capture of the ions (the lipid layer surface potential) and the logarithm of the ion concentration in the bulk solution, i.e., during sensor exposure to the analyte ${ }^{48,54}$. Here although the measurements were realized after drying the surface, we believe the double layer formed at the analyte/surface interface is preserved as it participates to stabilizing the interface and the Nernst equation still applies.

$$
\psi=2.3 \frac{k_{B} T}{Z e} \alpha \log [i o n]
$$

$\mathrm{z}$ is the charge carried by the ion, e, the electrical charge and $k_{B}$ and $\mathrm{T}$ the Boltzmann 


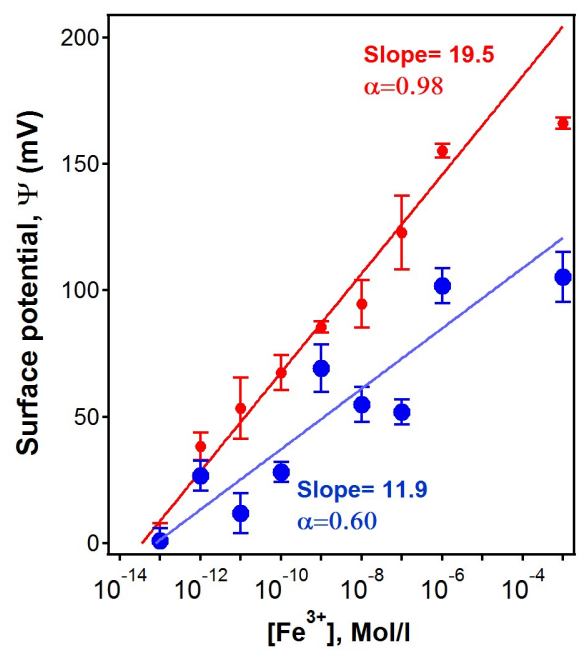

Figure 5: Lipid areas surface potential change after capture of $F e^{3}+$ ions using Array 1 (blue) and Array 3 (red) microarrays sensors. Both sensors display linear dependence with the logarithm of the ferric ions concentrations in the analyte solution for concentrations ranging from $1 \mathrm{pM}$ to $1 \mu \mathrm{M}$. Sensitivity factors $\alpha$ are determined from the curves slopes. For Array 1, a value of 0.6 is obtained suggesting low sensitivity. For Array 3 however $\alpha=0.98$, i.e. suggesting excellent performances of the sensor.

constant and the temperature. $\alpha$ is the sensitivity parameter which varies between 0 and 1 and which reflects the ratio between the surface buffer capacitance determined by the density of active chelators on the surface, and the electrolyte double layer capacitance. When $\alpha \sim 1$, the sensor approaches the Nernst limit. For trivalent ions, this value corresponds to a maximum slope of $19.8 \mathrm{mV} / \log [i o n]$. For the two types of sensors, the values of $\alpha$ were extracted from the slopes of the corresponding curves in Fig. 5. As expected for Array 1, a relatively low value of $\alpha$ of 0.6 is obtained reflecting the potential averaging. However for Array 3, a nearly perfect value of 0.98 is reached therefore indicating the excellent performances of our sensor.

\section{CONCLUSION}

In conclusion, we have presented herein the fabrication of an innovative microarray constituted of patterns of functional lipid monolayers and silicon that can be used as sensor using KPFM transducer. The technique takes advantage of the structure of the array with 
the silicon areas playing the role of reference for the measurement. Array dimensions are however important as the measured contact potential difference is the sum of all the capacitances between the tip/probe and the surface and can extend over a few microns. We have shown that with $1 \times 1 \mu^{2}$ arrays the measured CPD includes contributions of neighboring areas located outside the considered are. To discard this averaging effect, pattern size shall be higher than $3 \times 3 \mu \mathrm{m}^{2}$. Obviously this parameter has been determined for our tips, it is highly dependent on probes dimensions and shape and needs to be be determined for every system.

This work shows the potential of functional lipid microarrays as versatile generic sensing platforms. Use of cleaved lipids as building-block material allows the fabrication of an infinite range of lipid/probe complexes that can be spotted on the array using a spotter, and stabilized in air and overtime through the lipid polymerization process. This would create a multiplexed sensing platform constituted of different sensing areas dedicated to different ions. Ultimately such platform will allow the simultaneous multiplexing detection of a whole range of ions.

Demonstration was made here of the performances of such sensor with the detection of sub-picomolar concentrations of $\mathrm{Fe}^{3+}$ in solution using KPFM. Although the presented method has the advantage to be label free such use of platform could be extended to optical transduction methods.

\section{ACKNOWLEDGEMENTS}

This work was supported by the Centre National de la Recherche Scientifique and the Region PACA throughout its financial support (grants 2010-05815 DEB 10-1489 and 2010-12146 DEB 10-1174). We also thank F. Bedu, and I. Ozerov and the Nano-fabrication platform PLANETE CT-PACA. 


\section{Appendix A. Supplementary material}

1. Synthesis of $\gamma$-pyrone derivative as $\mathrm{Fe} 3+$ chelator and NMR characterization at every step of the synthesis. 2. Engineering of lipids with $\gamma$-pyrone and NMR characterization. 3. Specificity measurements.

\section{Graphical TOC entry}

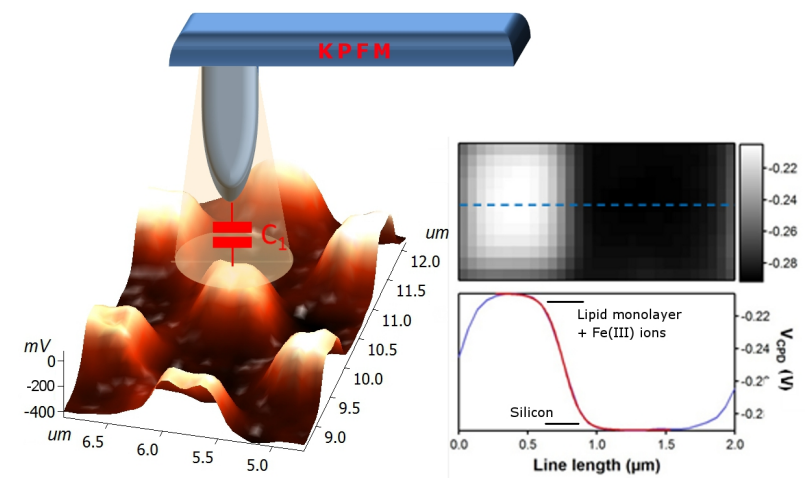

Figure 6: TOC

\section{REFERENCES}

\section{References}

(1) Kusi-Appiah, A. E.; Lowry, T. W.; Darrow, E. M.; Wilson, K. A.; Chadwick, B. P.; Davidson, M. W.; Lenhert, S. Lab on a Chip 2015, 15, 3397-3404.

(2) Lowry, T. W.; Prommapan, P.; Rainer, Q.; Van Winkle, D.; Lenhert, S. Sensors 2015, $15,20863$.

(3) Soga, N.; Watanabe, R.; Noji, H. Scientific reports 2015, 5. 
(4) Martinez, J. M. d. R.; Zaitseva, E.; Petersen, S.; Baaken, G.; Behrends, J. C. Small 2015, 11, 119-125.

(5) Sackmann, E. Science 1996, 271, 43-48.

(6) Tanaka, M.; Sackmann, E. Nature 2005, 437, 656-663.

(7) Basit, H.; Gaul, V.; Maher, S.; Forster, R. J.; Keyes, T. E. Analyst 2015, 140, 30123018.

(8) Orth, R.; Wu, M.; Holowka, D.; Craighead, H.; Baird, B. Langmuir 2003, 19, 15991605.

(9) Nair, P. M.; Salaita, K.; Petit, R. S.; Groves, J. T. Nature Protocols 2011, 6, 523-539.

(10) Li, J. S.; Ueda, E.; Nallapaneni, A.; Li, L. X.; Levkin, P. A. Langmuir 2012, 28, 8286-8291.

(11) Groves, J.; Boxer, S. Acc. Chem. Res. 2002, 35, 149-157.

(12) Han, C.-T.; Chao, L. Lab on a Chip 2015, 15, 86-93.

(13) Zhang, Y.; Wang, L.; Wang, X.; Qi, G.; Han, X. Chem. A: Eur. J. 2013, 19, 9059-9063.

(14) Hovis, J.; Boxer, S. Langmuir 2001, 17, 3400-3405.

(15) Yamada, M.; Imaishi, H.; Morigaki, K. Langmuir 2013, 29, 6404-6408.

(16) Jackson, B. L.; Groves, J. T. Langmuir 2007, 23, 2052-2057.

(17) Kung, L.; Groves, J.; Ulman, N.; Boxer, S. Adv. Mat. 2000, 12, 731+.

(18) Vats, K.; Kyoung, M.; Sheets, E. D. Biochim. Biophys. Acta-Biomembranes 2008, $1778,2461-2468$.

(19) Orth, R.; Kameoka, J.; Zipfel, W.; Ilic, B.; Webb, W.; Clark, T.; Craighead, H. Biophys. J. 2003, 85, 3066-3073. 
(20) Shaali, M.; Lara-Avila, S.; Dommersnes, P.; Ainla, A.; Kubatkin, S.; Jesorka, A. ACS Nano 2015, 9, 1271-1279.

(21) Foerste, A.; Pfirrmann, M.; Sachs, J.; Groeger, R.; Walheim, S.; Brinkmann, F.; Hirtz, M.; Fuchs, H.; Schimmel, T. Nanotechnology 2015, 26.

(22) Kalb, E.; Frey, S.; Tamm, L. Biochim. Biophys. Acta 1992, 1103, 307-316.

(23) Spinke, J.; Yang, J.; Wolf, H.; Liley, M.; Ringsdorf, H.; Knoll, W. Biophys. J. 1992, 63, 1667-1671.

(24) Kuhner, M.; Tampe, R.; Sackmann, E. Biophys. J. 1994, 67, 217-226.

(25) Arya, A.; Krull, U.; Thompson, M.; Wong, H. Anal. Chem. Acta 1985, 173, 331-336.

(26) Yoshikawa, K.; Hayashi, H.; Shimooka, T.; Terada, H.; Ishii, T. Biochem. Bioph. Res. Co. 1987, 145, 1092-1097.

(27) Scherer, M.; Scheibe, P.; Schoenhentz, J.; Hoffmann-Roeder, A.; Zentel, R. Colloid Polym. Sci. 2014, 292, 1803-1815.

(28) Peng, P.-Y.; Chiang, P.-C.; Chao, L. Langmuir 2015, 31, 3904-3911.

(29) Charrier, A.; Thibaudau, F. Biophys. J. 2005, 89, 1094-1101.

(30) Park, J.; McCormick, S. P.; Cockrell, A. L.; Chakrabarti, M.; Lindahl, P. A. Biochemistry 2014, 53, 3940-3951.

(31) Park, C.; Jang, K.; Lee, S.; You, J.; Lee, S.; Ha, H.; Yun, K.; Kim, J.; Lee, H.; Park, J.; Na, S. Nanotechnology 2015, 26.

(32) Charrier, A.; Mischki, T.; Lopinski, G. P. Langmuir 2010, 26, 2538-2543.

(33) Dumas, C.; El Zein, R.; Dallaporta, H.; Charrier, A. M. Langmuir 2011, 27, 1364313647. 
(34) Jacobs, H.; Leuchtmann, P.; Homan, O.; Stemmer, A. J. App. Phys. 1998, 84, 11681173.

(35) Davydovskaya, P.; Pentyala, V.; Yurchenko, O.; Hussein, L.; Pohle, R.; Urban, G. A. Sens. Actuator B-Chem. 2014, 193, 911-917, 17th International Conference on SolidState Sensors, Actuators and Microsystems, Barcelona, SPAIN, JUN 16-20, 2013.

(36) Oprea, A.; Barsan, N.; Weimar, U. Sens. Actuator B-Chem. 2009, 142, 470-493.

(37) Alexander, J.; Magonov, S.; Moeller, M. J. Vac. Sci. Tech. B 2009, 27, 903-911, 21st International Vacuum Nanoelectronics Conference, Wroclaw, POLAND, JUL 13-17, 2008.

(38) Nakamura, T.; Koyama, E.; Shimoi, Y.; Abe, S.; Ishida, T.; Tsukagoshi, K.; Mizutani, W.; Tokuhisa, H.; Kanesato, M.; Nakai, I.; Kondoh, H.; Ohta, T. J. Phys. Chem. B 2006, 110, 9195-9203.

(39) Kimura, M.; Horai, T.; Hanabusa, K.; Shirai, H. Adv. Mater. 1998, 10, 459+.

(40) Meier, M.; Schubert, U. Chem. Comm. 2005, 4610-4612.

(41) Abdizadeh, H.; Atilgan, A. R.; Atilgan, C. J. Biol. Inorg. Chem. 2015, 20, 705-718.

(42) Zhao, G.-Y.; Di, D.-H.; Wang, B.; Zhang, P.; Xu, Y.-J. Exp. Ther. Med. 2014, 8, 826-830.

(43) Millero, F.; Yao, W.; Aicher, J. arine Chem. 1995, 50, 21-39.

(44) Castellana, E. T.; Cremer, P. S. Surf. Sci. Rep. 2006, 61, 429-444.

(45) Watts, T.; Brian, A.; Kappler, J.; Marrack, P.; Mcconnell, H. PNAS 1984, 81, 75647568.

(46) El Zein, R.; Dallaporta, H.; Charrier, A. M. J. Phys. Chem. B 2012, 116, 7190-7195. 
(47) Nguyen, T. D.; El Zein, R.; Raimundo, J.-M.; Dallaporta, H.; Charrier, A. M. J. Mat. Chem. B 2013, 1, 443-446.

(48) Nguyen, T. D.; Labed, A.; El Zein, R.; Lavandier, S.; Bedu, F.; Ozerov, I.; Dallaporta, H.; Raimundo, J.-M.; Charrier, A. M. Biosens. Bioelectron. 2014, 54, 571-577.

(49) Mao, B.; Tao, Q.; Li, G. Meas. Sci. Tech. 2012, 23.

(50) Campiglio, P.; Campione, M.; Sassella, A. J. Phys. Chem. C 2009, 113, 8329-8335.

(51) Ichii, T.; Fukuma, T.; Kobayashi, K.; Yamada, H.; Matsushige, K. Nanotechnology 2004, 15, S30-S33, 6th International Conference on Non-contact Atomic Force Microscopy, Dingle, IRELAND, AUG 31-SEP 03, 2003.

(52) Palermo, V.; Palma, M.; Samori, P. Adv. Mater. 2006, 18, 145-164.

(53) McNally, H.; Janes, D.; Kasibhatla, B.; Kubiak, C. Supperlattices and Microstructures 2002, 31, 239-245.

(54) Knopfmacher, O.; Tarasov, A.; Wipf, M.; Fu, W.; Calame, M.; Schoenenberger, C. ChemPhysChem 2012, 13, 1157-1160. 